\title{
Usefulness of Ga-68 HBED-CC PSMA PET/CT for Tumor Staging in the Initial Diagnostic Assessment of Prostate Cancer
}

\section{Schreiter $\mathbf{V}^{1^{*}}$, Gericke $\mathbf{M}^{1}$, Beck $\mathbf{M}^{2}$, Ghadjar $\mathrm{P}^{2}$, Boening $\mathbf{G}^{1}$ and Schreiter $\mathrm{NF}^{3}$}

${ }^{1}$ Department of Radiology, Charité-Universitätsmedizin Berlin, Augustenburger Platz 1, 13353, Berlin, Germany

${ }^{2}$ Department of Radiation Oncology, Charité-Universitätsmedizin Berlin, Augustenburger Platz 1, 13353, Berlin, Germany

${ }^{3}$ Department of Nuclear Medicine, Charité-Universitätsmedizin Berlin, Augustenburger Platz 1, 13353, Berlin, Germany

\begin{abstract}
Purpose: To evaluate the identification of early prostate cancer by Ga-68 HBED-CC PSMA positron emission tomography/computed tomography (PET/CT) using the TNM classification for orientation.

Method: Sixteen men with a mean age of $68 \pm 6.8$ years (range, 55-80 years) who underwent Ga-68 HBED-CC PSMA PET/CT for initial diagnostic evaluation of suspected prostate cancer and biopsy at our department between August 2013 and June 2015 were included. Cancer extent determined by PET/CT and CT was retrospectively assessed by two independent observers $(\mathrm{O} 2, \mathrm{O} 3)$ without knowledge of clinical findings and compared with the initial diagnosis (O1). Histology of the prostate and of suspected metastatic lesions in conjunction with the interdisciplinary TNM diagnosis served as the gold standard.

Results: The TNM staging based on morphologic imaging criteria agreed with the gold standard for $01 / 02 / 03$ in $38 \% / 31 \% / 50 \%$ of cases for PET/CT and in $19 \% / 13 \% / 13 \%$ for CT. Detection rates for O1/O2/O3 were $38 / 44 \% / 56 \%$ versus $19 \% / 19 \% / 13 \%$ for T-stage (PET/CT versus $\mathrm{CT}$ ), $81 \% / 75 \% / 81 \%$ versus $75 \% / 63 \% / 75 \%$ for $\mathrm{N}$-stage, and $100 \% / 100 \% / 100 \%$ versus $100 \% / 88 \% / 100 \%$ for M-stage. Compared with histopathology, the TNM stage was underestimated with PET/CT (42\%) and even more so with CT alone (81\%).

Conclusion: Ga-68 HBED-CC PSMA PET/CT appears to be superior to CT alone in determining the TNM stage of prostate cancer, specifically with regard to the T-stage. Nevertheless, even Ga-68 HBED-CC PSMA PET/CT appears to be limited for characterization of the primary tumor and tends to underestimate the T-stage.
\end{abstract}

Keywords: Ga-68 HBED-CC PSMA PET/CT, Tumor staging, Prostate cancer

\section{Introduction}

With the advent of Ga-68 HBED-CC as a new tracer for positron emission tomography/computed tomography (PET/CT) of prostate cancer, this hybrid imaging modality has become a promising tool for whole-body evaluation of patients with prostate cancer what studies by Afshar-Oromieh et al. [1] and Sterzing et al. [2] have already shown. 68Ga HBED-CC is a urea-based inhibitor that binds specifically and with high affinity to the prostate-specific membrane antigen (PSMA) [3], a cell surface glycoprotein that is predominantly expressed in prostate cancer and prostate cancer metastases $[3,4]$. Initial clinical results with this tracer [5] show high detection rates in both patients with small recurrent prostate cancer lesions and patients with lo w prostate-specific antigen (PSA) levels [6]. Hence, this new tracer allows detection of early prostate cancer in situations where PET/CT with choline derivatives fails to identify any abnormalities $[5,7,8]$. This observation gives rise to the hope that Ga-68 HBED-CC may improve the detection of initial prostate cancer, which are not identified with PET/CT using choline derivatives as tracers [9]. Evaluation of the T-stage of prostate cancer is of particular interest here. This is because PET/CT using choline tracers, while allowing accurate assessment of the $\mathrm{N}$-stage and $\mathrm{M}$-stage, fails to provide adequate characterization of the primary tumor in the initial diagnostic assessment of prostate cancer [9]

In this study, we evaluate Ga-68 HBED-CC PSMA PET/CT for the initial diagnostic evaluation of prostate cancer using the TNM classification for orientation. The focus is on the characterization of the primary tumor to determine the benefit of this new tracer for prostate cancer staging in the initial diagnostic setting.

\section{Materials and Methods}

\section{Patients}

Between August 2013 and June 2015, a total of 385 men underwent PET/CT with Ga-68 HBED-CC, mostly for restaging of prostate cancer, less commonly for initial staging of suspected prostate cancer Our inclusion criteria were GA-68 HBED-CC performance for initial diagnosis of prostate cancer followed by biopsy of the prostate or of suspected metastasis from prostate cancer. 31 patients with suspected prostate cancer and an indication for Ga-68 HBED-CC PSMA PET/CT for initial diagnostic evaluation who subsequently underwent open or closed biopsy of the prostate or of suspected metastasis from prostate cancer fulfilled these inclusion criteria and were enrolled.

This study was approved by the ethical board of our institution. Our investigations were conducted in accordance with the Helsinki Declaration. All patients who were scanned in the PET/CT signed an informed consent.

*Corresponding author: Schreiter V, Department of Radiology, CharitéUniversitätsmedizin, Berlin, Germany, Tel: +49 30 450657165; E-mail: vera.schreiter@charite.de

Received April 12, 2016; Accepted June 02, 2016; Published June 06, 2016

Citation: Schreiter V, Gericke M, Beck M, Ghadjar P, Boening G, et al. (2016) Usefulness of Ga-68 HBED-CC PSMA PET/CT for Tumor Staging in the Initial Diagnostic Assessment of Prostate Cancer. J Nucl Med Radiat Ther 7: 291 doi:10.4172/2155-9619.1000291

Copyright: (c) 2016 Schreiter V, et al. This is an open-access article distributed under the terms of the Creative Commons Attribution License, which permits unrestricted use, distribution, and reproduction in any medium, provided the original author and source are credited. 
Citation: Schreiter V, Gericke M, Beck M, Ghadjar P, Boening G, et al. (2016) Usefulness of Ga-68 HBED-CC PSMA PET/CT for Tumor Staging in the Initial Diagnostic Assessment of Prostate Cancer. J Nucl Med Radiat Ther 7: 291. doi:10.4172/2155-9619.1000291

\section{PET/CT protocol}

The whole-body CT examination was performed immediately before PET acquisition. CT examinations were performed on a Philips Gemini TF ToF 16 scanner (Philips Medical Systems, Cleveland, USA) using the following dose parameters: $120 \mathrm{kV} ; 100-250 \mathrm{mAs}$ with dose modulation (D-DOM). Generally, the whole-body scan ranged from the mid-thigh to the skull base. The CT scan included in the PET/CT examination was acquired without contrast medium or, if indicated for whole-body staging, with $80 \mathrm{ml}$ to $120 \mathrm{ml}$ of Ultravist370 (Bayer HealthCare Pharmaceuticals, Berlin, Germany) injected as a bolus at a rate of $2 \mathrm{ml} / \mathrm{s}$ to $3 \mathrm{ml} / \mathrm{s}$. The amount of contrast agent was adjusted to body weight. A venous contrast phase (70 s after contrast administration) was acquired with $16 \mathrm{~mm} \times 1.5 \mathrm{~mm}$ reconstructed slice thickness and used for attenuation correction of the PET dataset. PET/CT was performed with Ga-68 HBED-CC PSMA ( $\left.={ }^{68} \mathrm{Ga}-\mathrm{DKFZ}-\mathrm{PSMA}-11\right)$. Synthesis of the tracer has been described in detail elsewhere [10]. The PET scan was acquired approx. $1 \mathrm{~h}$ after IV injection of $100 \mathrm{MBq}$ to $150 \mathrm{MBq}$ Ga-68 HBED-CC PSMA. Ten to twelve bed positions with $90 \mathrm{sec}$ per position were scanned with a $144 \times 144$ acquisition matrix and $576 \mathrm{~mm}$ field of view (FOV)

\section{Image analysis}

CT and PET/CT images were interpreted by a double-certified physician of nuclear medicine and radiology (O3) and a radiologist specialized in hybrid imaging $(\mathrm{O} 2)$. The results were compared with the initial diagnosis established by a physician of nuclear medicine $(\mathrm{O} 1)$. The experience of the three observers with this type of image analysis was more than 10 years $(\mathrm{O} 3)$, more than 6 years $(\mathrm{O} 2)$, and more than 8 years (O1). The readers were blinded and separately assessed the PET images, the CT images, and the PET/CT images. TNM stages were assigned on the basis of the metabolic information provided by $\mathrm{PET} / \mathrm{CT}$ and on the basis of the morphologic CT information. The 7th edition of the TNM classification of the UICC from 2009 was used for orientation [11].

\section{Final TNM classification}

The final TNM classification, which served as the gold standard, was determined by an interdisciplinary team consisting of pathologist, urologist, hematologist, radiotherapist, radiologist and physician of nuclear medicine. The interdisciplinary meeting was led by the patients' treating urologists, who made the final decision on further patient treatment based on the results of digital rectal examination, histology of tissue obtained by biopsy of the prostate, prostatectomy or suspicious metastatic lesions taking the patients' clinical data and available imaging findings into account. All specimens were examined at our department of pathology, blinded to PET/CT data. These were examined with hematoxylin-eosin stain to evaluate the Gleason grading after ISUP and pathological T and N stage [12]. Only for punch biopsy immunohistochemistry was used regularly. For complete prostatectomy specimens and lymph nodes, immunohistochemistry was only used in poorly differentiated carcinomas. Bone metastases were evaluated positive if there was a pathological tracer uptake in the PET or typical sclerotic lesions in the CT. The $7^{\text {th }}$ edition of the TNM classification of the UICC from 2009 was used.

\section{Statistical analysis}

The accuracy of TNM staging by CT and PET/CT was determined in relation to the gold standard as defined above. The T-, N-, and $\mathrm{M}$-stages determined with $\mathrm{CT}$ and $\mathrm{PET} / \mathrm{CT}$ were separately compared with the results of the final TNM classification. Statistical analysis was performed using Excel 2010 and SPSS 23.

\section{Results}

A total of 16 men with suspected prostate cancer who underwent Ga-68 HBED-CC PSMA PET/CT for initial diagnosis and subsequent open or closed biopsy were included in this retrospective analysis. They had a mean age of $68.00 \pm 6.8$ years (range, $55-80$ years). The mean PSA level at the time of PET/CT was $23.2 \mathrm{ng} / \mathrm{ml}$ (range, $4.4 \mathrm{ng} / \mathrm{ml}$ to 85.0 $\mathrm{ng} / \mathrm{ml}$ ). None of the patient was on androgen deprivation therapy at the time of PET/CT. The demographic and descriptive data evaluated at the time of PET/CT are summarized in Table 1.

The CT scan included in PET/CT was performed without contrast medium in 3 cases, while 13 patients were administered a contrast medium for a venous phase scan as described above. Two patients underwent closed prostate biopsy and 14 patients open prostate biopsy.

Stratified by imaging modality used for TNM classification, we found detection rates of $38 \%$ for the initial observer $(\mathrm{O} 1)$ and of $31 \%$ $(\mathrm{O} 2)$ and 50\% (O3) for Ga-68 HBED-CC PET/CT. For interpretation of CT alone, detection rates were $19 \%$ for the initial observer (O1) and $13 \%$ for $\mathrm{O} 2$ and $\mathrm{O} 3$. Table 2 summarizes detection rates including TNM classification stratified by T-, N- and M- stadium.

Compared with histology, the TNM stage was underestimated with PET/CT in $42 \%$ of cases and with CT in $81 \%$ of cases. The T-stage was underestimated with PET/CT in $44 \%$ of cases and with CT in $83 \%$ of cases. Stratified by observer, the results were as follows: O1 underestimated the T-stage in $56.3 \%$ of patients based on imaging morphology, $\mathrm{O} 2$ in $43.8 \%$ of patients, and $\mathrm{O} 3$ in $31.3 \%$ of patients. Overestimation of the T-stage on the basis of imaging morphology occurred in only $6.3 \%$ of patients for $\mathrm{O} 1$ versus $12.5 \%$ of patients for $\mathrm{O} 2$ and $\mathrm{O} 3$. Correlation of underestimation of T-stage on the basis of imaging morphology with a presumed reduction of maximum standard uptake values (SUVmax) of the suspicious prostate lesions revealed no significant association $(\mathrm{O} 1: \mathrm{p}=0.379 ; \mathrm{O} 2: \mathrm{p}=0.945, \mathrm{O} 3: \mathrm{p}=0.52)$. Metastases were not over- or underestimated with either modality. Figure 1 presents bar diagrams of

\begin{tabular}{|c|c|c|}
\hline & Mean (SD) & Median (range) \\
\hline Age in years & $68(6.8)$ & $69(55-80)$ \\
\hline PSA in $\mathbf{n g} / \mathbf{m l}$ & $23.2(24.7)$ & $10(4.4-85)$ \\
\hline Tracer in MBq & $113.1(17.0)$ & $110(79-144)$ \\
\hline Gleason score & $8.3(1.1)$ & $8(7-10)$ \\
\hline SUVmax in prostate & $14.6(8.8)$ & $14(2.6-34.9)$ \\
\hline
\end{tabular}

SUVmax: maximum standardized uptake value.

Table 1: Demographic and descriptive data of the 16 study patients.

\begin{tabular}{|c|c|c|c|}
\hline \multirow{2}{*}{$\mathbf{T}$} & Observer & PET/CT & $\mathbf{C T}$ \\
\hline \multirow{3}{*}{$\mathbf{N}$} & O1 & $38 \%$ & $19 \%$ \\
\cline { 2 - 4 } & O2 & $44 \%$ & $19 \%$ \\
\hline \multirow{3}{*}{$\mathbf{N}$} & O3 & $56 \%$ & $13 \%$ \\
\cline { 2 - 4 } & O1 & $81 \%$ & $81 \%$ \\
\hline \multirow{3}{*}{$\mathbf{M}$} & O2 & $75 \%$ & $63 \%$ \\
\hline \multirow{3}{*}{ TNM } & O3 & $81 \%$ & $75 \%$ \\
\hline & O1 & $100 \%$ & $100 \%$ \\
\cline { 2 - 4 } & O2 & $100 \%$ & $88 \%$ \\
\hline & O3 & $100 \%$ & $100 \%$ \\
\hline & O1 & $38 \%$ & $19 \%$ \\
\hline
\end{tabular}

Table 2: Detection rates of the three observers in correlation with the TNM stage. 
Citation: Schreiter V, Gericke M, Beck M, Ghadjar P, Boening G, et al. (2016) Usefulness of Ga-68 HBED-CC PSMA PET/CT for Tumor Staging in the Initial Diagnostic Assessment of Prostate Cancer. J Nucl Med Radiat Ther 7: 291. doi:10.4172/2155-9619.1000291
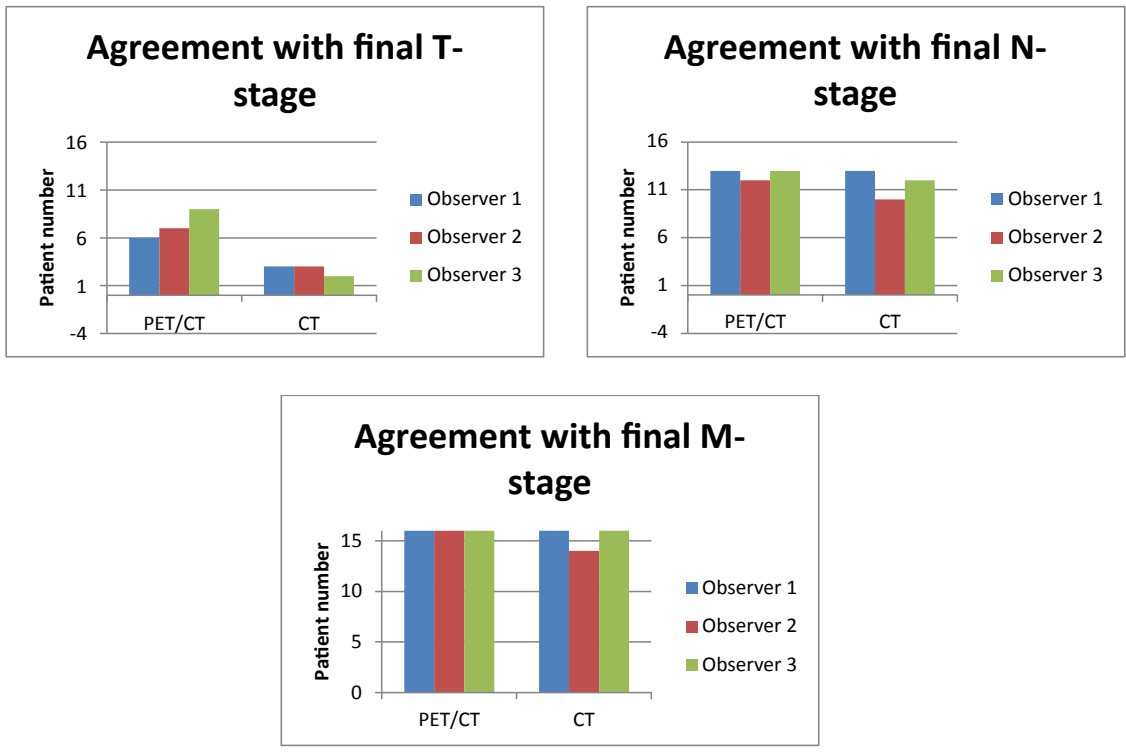

Figure 1: Bar diagrams of agreement of observers in the assessment of TNM stages using PET/CT and CT with the final TNM staging
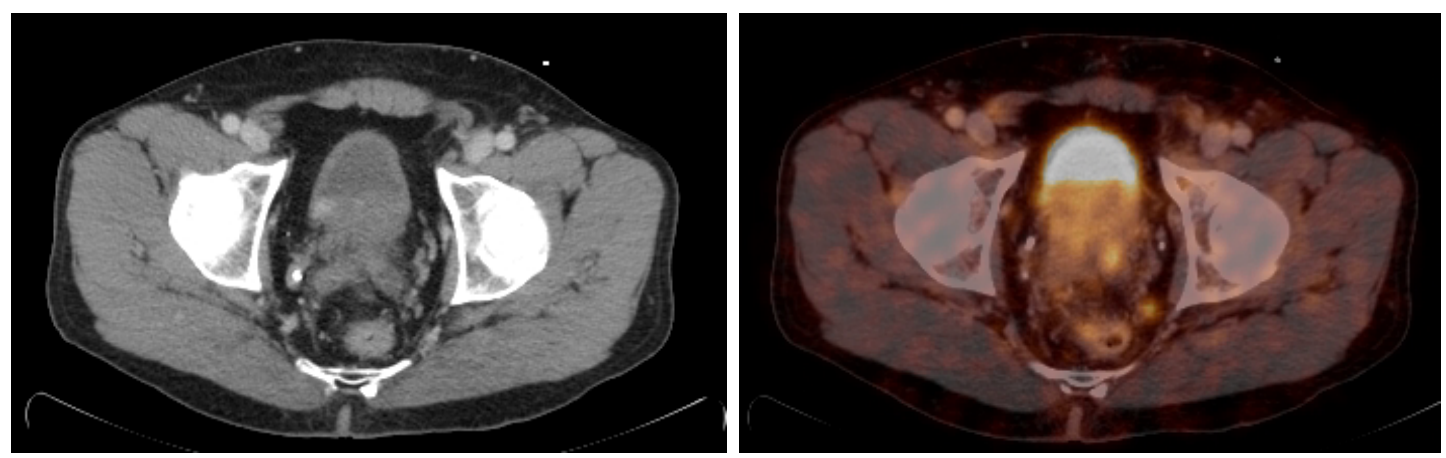

Figure 2: Consistent diagnosis of T3b prostate cancer with CT and Ga-68 HBED-CC PSMA PET/CT by all three observers

detection rates by observer and TNM stage. Figure 2 presents examples of images from a patient with consistent T-staging in CT and PET/CT by all three observers.

Ga-68 (HBED-CC) PSMA PET/CT for initial diagnosis yielded no evidence of suspicious lesions in one of the 16 patients. In another patient, Ga-68 HBED-CC PET/CT was inconclusive and repeat biopsy of the prostate was performed. In the remaining cases, tracer accumulation in the lesions suggested malignancy, and additional treatment was initiated based on these findings (Table 3 ).

Figure 3 presents an example of a para-aortic lymph node metastasis consistently identified by all three observers.

\section{Discussion}

PET/CT using Ga-68 HBED-CC PSMA is considered a promising new imaging tool for the diagnostic assessment of prostate cancer including metastatic spread as shown by Afshar-Oromieh et al. [1] in a large study cohort and Sterzing et al. [2], especially in patients with suspected recurrence. The study presented here was conducted to evaluate the use of Ga-68 HBED-CC PSMA PET/CT in the characterization of prostate cancer using the TNM stage determined by an interdisciplinary team and histopathology as the gold standard. In a study conducted by Sterzing et al. [2], PET/CT using the new tracer Ga-68 HBED- CC PSMA resulted in a modified TNM staging with a change in treatment in $50.8 \%$ of the patients examined. To the best of our knowledge, this is the first study of Ga-68 HBED-CC PSMA $\mathrm{PET} / \mathrm{CT}$ in which this modality is investigated by directly evaluating the TNM stage determined on the basis of imaging morphology and metabolic in correlation with the TNM stage determined by an interdisciplinary team using all information available including histopathology. Agreement of PET/CT with this standard ranged from $38 \%$ to $50 \%$ for the three observers. In the separate analysis of the results for the primary tumor, lymph node involvement, and metastasis, the accuracy of Ga-68 HBED-CC PSMA PET/CT for characterization of the T-stage $(38 / 44 \% / 56 \%$ for $\mathrm{O} 1 / \mathrm{O} 2 / \mathrm{O} 3)$ is lower than for the $\mathrm{N}$-stage or M-stage $(81 \% / 75 \% / 81 \%$ and $100 \% / 100 \% / 100 \%$ for $\mathrm{O} 1 / \mathrm{O} 2 / \mathrm{O} 3)$. The T-stage tends to be underestimated based on imaging information with CT and with Ga-68 HBED-CC PSMA PET/CT. Underestimation might be due to the poor detection rates and a lack of soft tissue contrast of the CT in initial staging of primary cancer of the prostate [13]. Whilst Ga-68 HBED-CC PSMA PET/CT has better detection rates than CT but still lack from the same soft tissue contrast as the $\mathrm{CT}$ which makes it difficult to distinguish the subcategory of each T-stage in the prostate 
Citation: Schreiter V, Gericke M, Beck M, Ghadjar P, Boening G, et al. (2016) Usefulness of Ga-68 HBED-CC PSMA PET/CT for Tumor Staging in the Initial Diagnostic Assessment of Prostate Cancer. J Nucl Med Radiat Ther 7: 291. doi:10.4172/2155-9619.1000291

Page 4 of 5

\begin{tabular}{|c|c|c|c|c|c|}
\hline Patient No. & $\begin{array}{l}\text { Initiation of treatment after Ga-68 } \\
\text { HBED-CC PSMA PET/CT (Y-yes/X-no) }\end{array}$ & $\begin{array}{c}\text { Radical prostatovesiculectomy } \\
\text { (Y-yes/X-no) }\end{array}$ & $\begin{array}{l}\text { Bilateral lymphadenectomy } \\
\text { (date) }\end{array}$ & Final TNM & $\begin{array}{l}\text { Additionaly initiated } \\
\text { treatment }\end{array}$ \\
\hline 1 & $x$ & - & - & TONOMO & - \\
\hline 2 & $x$ & - & - & TONOMO & $\begin{array}{l}\text { Repeat biopsy negative } \\
(06 / 14)\end{array}$ \\
\hline 3 & $\checkmark$ & $\checkmark$ & $\checkmark(04 / 14)$ & T3bNOMO & - \\
\hline 4 & $\checkmark$ & $\checkmark$ & $\checkmark(05 / 14)$ & T2cNOMO & - \\
\hline 5 & $\checkmark$ & $\checkmark$ & $\checkmark(06 / 14)$ & T3bN1M1b & $\begin{array}{c}\text { Enantone since } 07 / 2014 \\
\text { Bicalutamide since } \\
11 / 2014\end{array}$ \\
\hline 6 & $\checkmark$ & $\checkmark$ & $\checkmark(07 / 14)$ & T3bN1M1b & $\begin{array}{c}\text { Cyberknife of metastasis } \\
\text { in left acetabulum, } 18 \mathrm{~Gy}, \\
02 / 07 / 14\end{array}$ \\
\hline 7 & $\checkmark$ & $\checkmark$ & $\checkmark(09 / 15)$ & T3bN1M0 & $\begin{array}{c}\text { Zoladex since 09/2014 } \\
\text { Salvage radiotherapy, } 66 \\
\text { Gy, 11/2014- 02/2015 }\end{array}$ \\
\hline 8 & $\checkmark$ & $\checkmark$ & $\checkmark(03 / 15)$ & T3aN1M0 & - \\
\hline 9 & $\checkmark$ & $\checkmark$ & $\checkmark(01 / 15)$ & T3bN1M0 & - \\
\hline 10 & $\checkmark$ & $\checkmark$ & $\checkmark(04 / 15)$ & T2cNOMO & - \\
\hline 11 & $\checkmark$ & $\checkmark$ & $\checkmark(07 / 15)$ & T2bNOMO & - \\
\hline 12 & $\checkmark$ & $\checkmark$ & $\checkmark(05 / 15)$ & T3bN1M0 & - \\
\hline 13 & $\checkmark$ & $\checkmark$ & $\checkmark(06 / 15)$ & T2cNOMO & - \\
\hline 14 & $\checkmark$ & $\checkmark$ & $\checkmark(06 / 15)$ & T3bN1M0 & $\begin{array}{l}\text { Salvage radiotherapy } \\
\text { (since } 11 / 2015,70.3 \mathrm{~Gy})\end{array}$ \\
\hline 15 & $\checkmark$ & $\checkmark$ & $\checkmark(07 / 14)$ & T2cNOMO & - \\
\hline 16 & $\checkmark$ & $\checkmark$ & $\checkmark(08 / 15)$ & T2cNOMO & - \\
\hline
\end{tabular}

Table 3: Treatment initiated after demonstration of suspicious lesions by initial Ga-68 HBED-CC PET/CT.
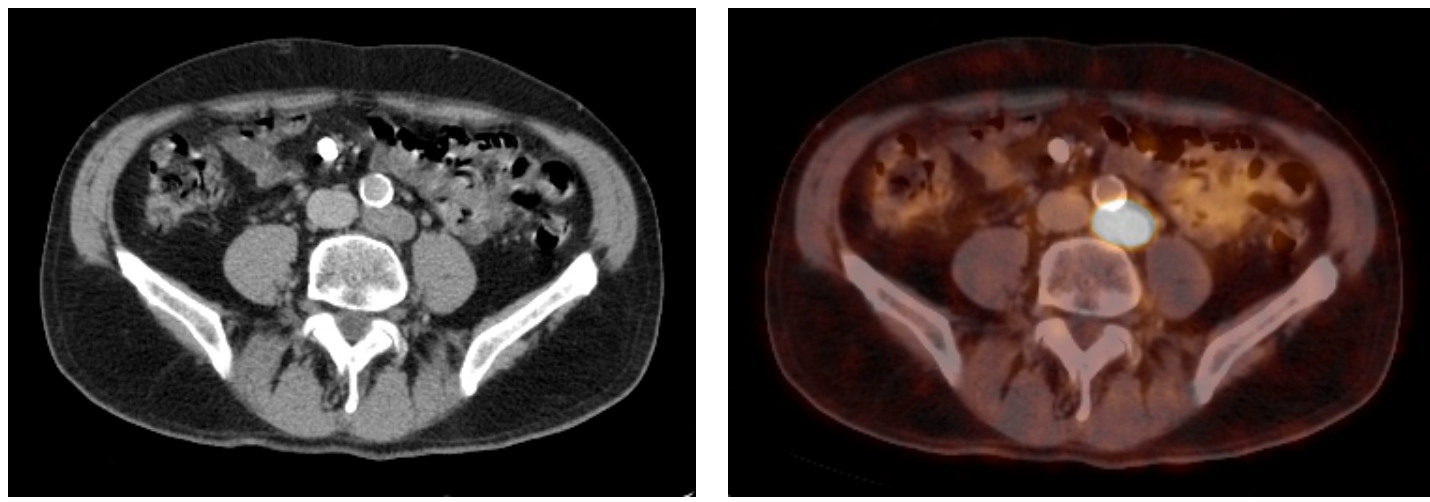

Figure 3: 75-year-old patient with para-aortic lymph node metastasis, which was suspected as a metastasis by all three observers in both CT alone and Ga-68 HBED-CC PSMA PET/CT.

properly. Another for underestimated T-stage in prostate cancer could be due to small tumor mass or micro metastasis do not show enough tracer uptake to be properly detected by PET/CT.

These findings of our study suggest that MRI, with its highresolution, cannot be replaced, especially for T-staging, and thus continues to have a role in the diagnostic assessment of prostate cancer - either when used alone or when used in combination with another modality in fusion imaging. In recent years, prostate cancer MRI has developed from the use of endorectal coil techniques with accuracies, sensitivities, and positive predictive values (PPV) of $79.8 \%, 85.3 \%$, and $92.6 \%$ for cancers larger than $1 \mathrm{~cm}$ and of $24.2 \%, 26.2 \%$, and $75.9 \%$ for cancers less than $1 \mathrm{~cm}$ in diameter and an accuracy of $68 \%$ for the differentiation of $\mathrm{T} 2$ and $\mathrm{T} 3$ prostate cancer to the use of body coils and multiparametric pulse sequences $[14,15]$. Multiparametric imaging offers the advantage of providing valid additive information on cancer extent outside the prostate, as for instance shown in a prospective study of Lista et al. [16]. In this study of 85 patients, MRI had 33\% sensitivity and $96 \%$ specificity in the characterization of extracapsular cancer manifestation versus $58 \%$ and $98 \%$ for multiparametric MRI [16]. In the assessment of the T-stage of prostate cancer, multiparametric MRI with use of a fully automated computer system has been reported to have a median sensitivity of $84 \%$ ( $1^{\text {st }}$ to $3^{\text {rd }}$ quartile, $77 \%$ to $93 \%$ ) and a median specificity of $86 \%$ ( $1^{\text {st }}$ to $3^{\text {rd }}$ quartile, $76 \%$ to $95 \%$ ) and a lesion-based sensitivity of $96 \%$ [17]. Studies comparing MRI with PET/CT in the characterization of primary prostate cancer show better sensitivity for MRI with use of diffusion-weighted imaging (MRI) and determination of the apparent diffusion coefficient (ADC). A case in point is the study of Rowe et al. [18], who evaluated the diagnostic accuracy of MRI and 18F-DCFBC PET in the detection and characterization of prostate cancer. This study found 39\% sensitivity, $89 \%$ specificity, a PPV of $73 \%$ and an NPV of $58 \%$ and $65 \%$ accuracy for MRI versus $17 \%$ sensitivity, $96 \%$ specificity, $81 \%$ PPV, $53 \%$ NPV, and $57 \%$ accuracy for 18 F-DCFBC 
Citation: Schreiter V, Gericke M, Beck M, Ghadjar P, Boening G, et al. (2016) Usefulness of Ga-68 HBED-CC PSMA PET/CT for Tumor Staging in the Initial Diagnostic Assessment of Prostate Cancer. J Nucl Med Radiat Ther 7: 291. doi:10.4172/2155-9619.1000291

PET [18]. The combination of MRI and PET/CT information might be of particular interest for planning radiotherapy of prostate cancer in the setting of personalized treatment approaches in the future [19]. In the evaluation of the dominant intraprostatic lesion, Zamboglou et al. [20] found 47\% agreement between multiparametric MRI and Ga-68 HBED-CC PSMA PET/CT in correlation with histopathology.

This study has some limitations such as a small patient number. The M-stage of prostate cancer could not be verified clearly in this study due to no existence of reference for this stage.

In summary, our results show that PET/CT with Ga-68 HBEDCC PSMA is clearly superior to CT alone for TNM staging in the initial diagnostic evaluation of prostate cancer. Correct assessment of the tumor appears to pose the greatest challenge to Ga-68 HBEDCC PSMA PET/CT, which tends to underestimate the T-stage. MRI has advantages in T-staging and thus continues to have a role in the diagnostic assessment of prostate cancer.

\section{Acknowledgement}

Dr. V. Schreiter is participant in the Charité Clinical Scientist Program funded by the Charité Universitätsmedizin Berlin and the Berlin Institute of Health.

\section{References}

1. Afshar-Oromieh A, Avtzi E, Giesel FL, Holland-Letz T, Linhart HG, et al. (2015) The diagnostic value of PET/CT imaging with the (68)Ga-labelled PSMA ligand HBED-CC in the diagnosis of recurrent prostate cancer. Eur J Nucl Med Mol Imaging 42: 197-209.

2. Sterzing F, Kratochwil C, Fiedler H (2016) (68)Ga-PSMA-11 PET/CT: a new technique with high potential for the radiotherapeutic management of prostate cancer patients. Eur J Nucl Med Mol Imaging 43: 34-41.

3. Silver DA, Pellicer I, Fair WR, Heston WD, Cordon-Cardo C (1997) Prostatespecific membrane antigen expression in normal and malignant human tissues. Clin Cancer Res 3: 81-85.

4. Murphy GP, Elgamal AA, Su SL, Bostwick DG, Holmes EH (1998) Current evaluation of the tissue localization and diagnostic utility of prostate specific membrane antigen. Cancer 83: 2259-2269.

5. Afshar-Oromieh A, Haberkorn U, Eder M, Eisenhut M, Zechmann CM (2012) [68Ga]Gallium-labelled PSMA ligand as superior PET tracer for the diagnosis of prostate cancer: comparison with 18F-FECH. Eur J Nucl Med Mol Imaging 39: 1085-1086.

6. Eder M, Eisenhut M, Babich J, Haberkorn U (2013) PSMA as a target for radiolabelled small molecules. Eur J Nucl Med Mol Imaging 40: 819-823.

7. Igerc I, Kohlfürst S, Gallowitsch HJ, Matschnig S, Kresnik E, et al. (2008) The value of $18 \mathrm{~F}$-choline PET/CT in patients with elevated PSA-level and negative prostate needle biopsy for localisation of prostate cancer. Eur J Nucl Med Mol Imaging 35: 976-983.

8. Morigi JJ, Stricker PD, van Leeuwen PJ, Tang R, Ho B, et al. (2015) Prospective Comparison of 18F-Fluoromethylcholine Versus 68Ga-PSMA PET/CT in Prostate Cancer Patients Who Have Rising PSA After Curative Treatment and Are Being Considered for Targeted Therapy. J Nucl Med 56: 1185-1190.

9. Picchio M, Mapelli P, Panebianco V, Castellucci P, Incerti E, et al. (2015) Imaging biomarkers in prostate cancer: role of PET/CT and MRI. Eur J Nucl Med Mol Imaging 42: 644-655.

10. Eder M, Neels O, Müller M, Bauder-Wüst U, Remde $Y$, et al. (2014) Nove Preclinical and Radiopharmaceutical Aspects of [68Ga]Ga-PSMA-HBED-CC: A New PET Tracer for Imaging of Prostate Cancer. Pharmaceuticals (Basel) 7: 779-796.

11. Sobin LH, Gospodarowicz MK, Wittekind C (2009) TNM Classification of Malignant Tumours $\left(7^{\text {th }}\right.$ Edn), Oxford, Wiley-Blackwell.

12. Epstein JI, Allsbrook WC, Amin MB, Egevad LL, Committee IG (2005) The 2005 International Society of Urological Pathology (ISUP) Consensus Conference on Gleason Grading of Prostatic Carcinoma. Am J Surg Pathol 29: 1228-1242.

13. Engeler CE, Wasserman NF, Zhang G (1992) Preoperative assessment of prostatic carcinoma by computerized tomography. Weaknesses and new perspectives. Urology 40: 346-350.

14. Jager GJ, Ruijter ET, van de Kaa CA, de la Rosette JJ, Oosterhof GO, et al. (1996) Local staging of prostate cancer with endorectal MR imaging: correlation with histopathology. AJR Am J Roentgenol 166: 845-852.

15. Nakashima J, Tanimoto A, Imai Y, Mukai M, Horiguchi Y, et al. (2004) Endorecta MRI for prediction of tumor site, tumor size, and local extension of prostate cancer. Urology 64: 101-105.

16. Lista F, Gimbernat H, Cáceres F, Rodríguez-Barbero JM, Castillo E, et al. (2014) Multiparametric magnetic resonance imaging for the assessment of extracapsular invasion and other staging parameters in patients with prostate cancer candidates for radical prostatectomy. Actas Urol Esp 38: 290-297.

17. Giannini V, Mazzetti S, Vignati A, Russo F, Bollito E, et al. (2015) A fully automatic computer aided diagnosis system for peripheral zone prostate cancer detection using multi-parametric magnetic resonance imaging. Comput Med Imaging Graph 46: 219-226.

18. Rowe SP, Gage KL, Faraj SF, Macura KJ, Cornish TC, et al. (2015) ${ }^{18} \mathrm{~F}-\mathrm{DCFBC}$ $\mathrm{PET} / \mathrm{CT}$ for PSMA-Based Detection and Characterization of Primary Prostate Cancer. J Nucl Med 56: 1003-1010.

19. Eiber M, Weirich G, Holzapfel K, Souvatzoglou M, Haller B, et al. (2016) Simultaneous 68Ga-PSMA HBED-CC PET/MRI Improves the Localization of Primary Prostate Cancer. Eur Urol S0302-2838(16)00011-7.

20. Zamboglou C, Wieser G, Hennies S, Rempel I, et al. (2016) MRI versus (68) Ga-PSMA PET/CT for gross tumour volume delineation in radiation treatment planning of primary prostate cancer. Eur J Nucl Med Mol Imaging 43: 889-897. 